\title{
Spin correlations in the paramagnetic phase and ring exchange in $\mathrm{La}_{2} \mathrm{CuO}_{4}$
}

\author{
A.M. Toader ${ }^{1}$, J.P. Goff ${ }^{1}$, M. Roger ${ }^{2}$, N. Shannon ${ }^{2}$, J.R. Stewart ${ }^{3}$ and M. Enderle ${ }^{3}$ \\ 1 Department of Physics, University of Liverpool, \\ Oliver Lodge Laboratory, Liverpool L69 7ZE, United Kingdom. \\ 2 Service de Physique de l'Etat Condensé, Commissariat à l'Energie Atomique, \\ Centre d'Etudes de Saclay, 91191 Gif sur Yvette Cedex, France. \\ 3 Institut Laue-Langevin,156X, 38402 Grenoble Cedex, France.
}

(Dated: September 12, 2018)

\begin{abstract}
Spin correlations in the paramagnetic phase of $\mathrm{La}_{2} \mathrm{CuO}_{4}$ have been studied using polarized neutron scattering, with two important results. First, the temperature dependence of the characteristic energy scale of the fluctuations and the amplitude of the neutron structure factor are shown to be in quantitative agreement with the predictions of the quantum non-linear sigma model. Secondly, comparison of a high-temperature series expansion of the equal-time spin correlations with the diffuse neutron intensity provides definitive experimental evidence for ring exchange.
\end{abstract}

Heisenberg was the first to realize that strong effective spin interactions arise from the principle of the indistinguishability of the particles[1]. Dirac generalized this concept, in the context of Group Theory, to include higher order interactions [2]. Multi-particle exchange dominates the physics of the quantum solid ${ }^{3} \mathrm{He} 3$ but, surprisingly, is generally not taken into account for electronic magnetic materials. The most powerful technique for exploring exchange interactions is the study of excitations from the ordered phase using inelastic neutron scattering. However, ambiguities in the interpretation of magnon dispersion curves sometimes mean that higher order terms remain hidden. By employing the independent approach of studying the instantaneous spin correlations in the paramagnetic phase, we obtain complementary information that enables a better understanding of the exchange mechanism. In this letter we describe studies of the diffuse magnetic scattering from $\mathrm{La}_{2} \mathrm{CuO}_{4}$ which provide compelling, quantitative evidence for the existence of four-particle cyclic exchange.

$\mathrm{La}_{2} \mathrm{CuO}_{4}$ is of great intrinsic interest both as the parent compound of a canonical high-temperature superconductor, and as a very good realization of a two-dimensional quantum Heisenberg antiferromagnet (2DQHAF). Magnetic Raman experiments [4], infrared absorption studies 5, 6] and inelastic neutron scattering measurements [] show definitively the inadequacy of the nearest-neighbour Heisenberg model, and suggest the possibility that four-particle exchange may be significant.

In an important series of experiments to study the diffuse magnetic scattering from $\mathrm{La}_{2} \mathrm{CuO}_{4}$ using unpolarized neutrons [8] the temperature dependence of the magnetic correlation length was found to agree with the predictions of the quantum non-linear sigma model $(\mathrm{QNL} \sigma \mathrm{M})[9]$. However, the observed amplitude shows dramatic deviations from the predictions of this theory [8]. The QNL $\sigma \mathrm{M}$ is the simplest possible effective action for a 2DQHAF that is compatible with the long-wavelength spin waves and that does not assume a spontaneously broken symmetry. Moreover, its pre- dictions should hold even in the presence of four-spin exchange, as discussed below. Here we study the dynamical spin correlations above the Néel temperature using polarized neutrons, and find complete agreement of the observed 2D critical fluctuations with the predictions of the QNL $\sigma \mathrm{M}$.

Dirac's approach provides the most transparent theoretical framework to examine higher-order exchange interactions [2]. His analysis leads to an effective spin Hamiltonian

$$
\mathcal{H}_{e f f}=-\sum_{\lambda}(-1)^{p_{\lambda}} J_{\lambda} \mathcal{P}_{\lambda}^{\sigma}
$$

where $\lambda$ runs over all possible permutations of spin $\mathcal{P}_{\lambda}^{\sigma}$ within the symmetric group, $J_{\lambda}$ is the exchange energy associated with a given permutation and $p_{\lambda}$ its parity. Any permutation can be expressed in terms of cyclic exchange processes. Thouless was the first to point out that cyclic permutations of an even number of spins lead to $\mathrm{AF}$ exchange, whereas when an odd number of spins are permuted the resulting interaction is FM[10]. Only the values of the exchange parameters $J_{\lambda}$ depend on the choice of model; the form of interaction between spins is quite general.

In $\mathrm{La}_{2} \mathrm{CuO}_{4}$, retaining the most important exchange processes involved in a plaquette, the general effective spin Hamiltonian is given by:

$$
\begin{gathered}
\mathcal{H}_{\text {eff }}=J_{2}^{(1)} \sum_{<i j>}^{(1)} \mathcal{P}_{i j}^{\sigma}+J_{2}^{(2)} \sum_{<i j>}^{(2)} \mathcal{P}_{i j}^{\sigma}+J_{2}^{(3)} \sum_{<i j>}^{(3)} \mathcal{P}_{i j}^{\sigma} \\
-J_{3} \sum_{i j k}\left[\mathcal{P}_{i j k}^{\sigma}+\left(\mathcal{P}_{i j k}^{\sigma}\right)^{-1}\right]+J_{4} \sum_{i j k l}\left[\mathcal{P}_{i j k l}^{\sigma}+\left(\mathcal{P}_{i j k l}^{\sigma}\right)^{-1}\right](2)
\end{gathered}
$$

the $J_{2}^{(n)}$ are pair-exchange frequencies between nearest (1), next-nearest (2) and next-next nearest neighbours (3), $J_{3}$ and $J_{4}$ represent three-and four-particle cyclic exchanges in a plaquette. In terms of spin operators

$$
\mathcal{H}_{\text {eff }}=\left(2 J_{2}^{(1)}-8 J_{3}+2 J_{4}\right) \sum_{<i j>}^{(1)} \mathbf{S}_{i} \cdot \mathbf{S}_{j}
$$




$$
\begin{array}{r}
+\left(2 J_{2}^{(2)}-4 J_{3}+J_{4}\right) \sum_{<i j>}^{(2)} \mathbf{S}_{i} \cdot \mathbf{S}_{j}+2 J_{2}^{(3)} \sum_{<i j>}^{(3)} \mathbf{S}_{i} \cdot \mathbf{S}_{j} \\
+4 J_{4} \sum_{<i j k l>}\left[\left(\mathbf{S}_{i} \cdot \mathbf{S}_{j}\right)\left(\mathbf{S}_{k} \cdot \mathbf{S}_{l}\right)+\left(\mathbf{S}_{j} \cdot \mathbf{S}_{k}\right)\left(\mathbf{S}_{l} \cdot \mathbf{S}_{i}\right)\right. \\
\left.-\left(\mathbf{S}_{i} \cdot \mathbf{S}_{k}\right)\left(\mathbf{S}_{j} \cdot \mathbf{S}_{l}\right)\right]
\end{array}
$$

We note that the four-particle cyclic exchange $J_{4}$ in Eq. (2) contributes both four-spin and two-spin terms to Eq. (3). On a square lattice, with the two-sublattice antiferromagnetic Néel phase there is a remarkable (although fortuitous) exact cancellation of all contributions of the $J_{4}$ terms in linear spin-wave theory. This means that all quantities (in particular the magnon dispersion) calculated within this simple framework are the same as those corresponding to the pure Heisenberg Hamiltonian

$$
H_{\text {Heis }}=\sum_{n=1}^{n=3} 2 \tilde{J}_{2}^{(n)} \sum_{<i j>}^{(n)} \mathbf{S}_{i} \cdot \mathbf{S}_{j}
$$

with $\tilde{J}_{2}^{(1)}=J_{2}^{(1)}-4 J_{3}, \tilde{J}_{2}^{(2)}=J_{2}^{(2)}-2 J_{3}$ and $\tilde{J}_{2}^{(3)}=J_{2}^{(3)}$, and are completely blind to the four-particle permutation term $J_{4}$.

For simplicity, we can model the $\mathrm{Cu}-\mathrm{O}$ planes in $\mathrm{La}_{2} \mathrm{CuO}_{4}$ using the half-filled one-band Hubbard model

$$
H=-t \sum_{i j \sigma} c_{i \sigma}^{+} c_{j \sigma}+U \sum_{i} n_{\uparrow} n_{\downarrow}
$$

where the hopping energy $t$ characterizes the kinetic energy, the potential energy $U \gg t$ is the penalty for double occupancy, $c\left(c^{+}\right)$are the annihilation (creation) operators and $n=c^{+} c$ is a number operator. At fourth order in a $\kappa=t / U$ expansion, the $J_{\lambda}$ 's appear as $J_{2}^{(1)} / U=2 \kappa^{2}\left(1+4 \kappa^{2}\right), J_{2}^{(2)} / U=12 \kappa^{4}, J_{2}^{(3)} / U=2 \kappa^{4}$, $J_{3} / U=10 \kappa^{4}$ and $J_{4} / U=20 \kappa^{4}[11$, 12]. More intricate expressions are obtained for a more general three-band Hubbard model [13]. The effective interaction between next-nearest neigbour pairs $\tilde{J}_{2}^{(2)}$ becomes negative (i.e. FM) because of the presence of the FM three-particle term $J_{3}$. The next-next-nearest neighbour term is small and can be neglected. The magnitude of the four-particle cyclic exchange $J_{4}$ is large.

Since spin waves are insensitive to four-particle cyclic exchange, the curvature of the magnon dispersion at the zone boundary [7] is instead entirely due to the ferromagnetic effective next-nearest neighbour exchange $\tilde{J}_{2}^{(2)}$. In contrast, there is no such cancellation of the four-spin term for the static susceptibility at high temperatures. We have, therefore, studied the diffuse scattering in the paramagnetic phase, and this is a new approach to the investigation of higher order exchange. The dynamical structure factor for neutron scattering is given by

$$
S(\mathbf{Q}, \omega)=\frac{\omega}{1-\mathrm{e}^{-\omega / T}} \frac{S(0)}{1+(q \xi)^{2}} \times
$$

$$
\left[\frac{\Gamma}{(\omega-c q)^{2}+\Gamma^{2}}+\frac{\Gamma}{(\omega+c q)^{2}+\Gamma^{2}}\right]
$$

where $\Gamma$ is the characteristic energy. Integration over energy transfer yields information on the equal-time spinspin correlations since

$$
\int_{-\infty}^{\infty} S(\mathbf{Q}, \omega) d \omega \approx \sum_{i} \mathrm{e}^{\imath \mathbf{Q} \cdot \mathbf{R}_{i}}\left\langle S_{i}^{z} S_{0}^{z}\right\rangle \approx T \chi(\mathbf{Q})
$$

The wave-vector dependent static susceptibility $\chi(\mathbf{Q})$ can be calculated from the exchange energies using a hightemperature series expansion.

A $2 \mathrm{~g}$ single-crystal of $\mathrm{La}_{2} \mathrm{CuO}_{4}$ from the array used to study the spin waves in the ordered phase [7] was mounted inside furnaces, and the diffuse magnetic scattering was measured in the temperature range $300-500 \mathrm{~K}$ using the polarized neutron spectrometers D7 and IN20 at the Institut Laue-Langevin. $X Y Z$ polarization analysis was employed to separate the magnetic signal from the coherent structural and spin-incoherent backgrounds 14. The scattering intensity measured in the $(h, 0, l)$ plane at room temperature using the multidetector on D7 is presented in Fig. 1(a) for the nuclear scattering showing the structural Bragg reflections and (b) for the purely magnetic signal showing the appearance of a rod of intensity perpendicular to the cuprate planes. The integrated intensity along the $\mathbf{Q}^{3 D}=(1,0, l)$ rod for $\mathrm{La}_{2} \mathrm{CuO}_{4}$ was measured as a function of temperature with the incident wave vector fixed, $k_{i}=2.08 \AA^{-1}$, and the final wave vector parallel to the normal to the cuprate planes in a similar manner to Ref. 8] so that the cuprate square-lattice wave vector remained fixed at $\mathbf{Q}^{2 D}=\left(\frac{1}{2}, \frac{1}{2}\right)$ for all energy transfers. For a quantitative temperature dependence of the intensity integrated over energy transfer it is essential to determine how the spectral line shape varies with temperature. Energy scans were performed with $\mathbf{Q}$ fixed using the triple-axis spectrometer IN20 and typical spectra are presented in Fig. 2(a).

In the $\mathrm{QNL} \sigma \mathrm{M}$, the correlation length $\xi$ is given by $[\underline{9}$

$$
\xi(T)=C_{\xi}\left[\frac{\hbar v_{s}}{\rho_{s}}\right] \exp \left[\frac{2 \pi \rho_{s}}{k_{B} T}\right]
$$

and the energy width $\Gamma$ is related to $\xi$ by

$$
\Gamma=C_{\Gamma} v_{s}\left[\frac{T}{2 \pi \rho_{s}}\right]^{\frac{1}{2}} \frac{1}{\bar{\xi}}
$$

where $v_{s}$ is the spin-wave velocity, $\rho_{s}$ is the spin stiffness and $C_{\xi}$ and $C_{\Gamma}$ are undetermined constants of order one. Figure 2(b) compares the temperature dependence of $\xi$ deduced from equation (9) using the values of $\Gamma$ determined in energy scans. There is excellent agreement between the dynamical predictions of the QNL $\sigma \mathrm{M}$ and the correlation lengths measured using unpolarized neutrons by Birgeneau et al. [8]. The intensities measured in fixed-Q energy scans on IN20 integrated over 
energy transfer were converted to the amplitude $\mathrm{S}(0)$ in equation (6) using the known correlation lengths [8]. The intensities measured on D7 without energy analysis were corrected using the spectral line shapes extrapolated from Fig. 2(b), the instrumental energy window, the $\mathrm{Cu}^{2+}$ magnetic form factor and the correlation lengths, and the amplitudes from both experiments are combined in Fig. [3] The leading term in the expression for the ratio of the amplitude to the correlation length squared in the QNL $\sigma \mathrm{M}$ is 9

$$
\frac{S(0)}{\xi^{2}} \approx\left(\frac{k_{B} T}{2 \pi \rho_{s}}\right)^{2}
$$

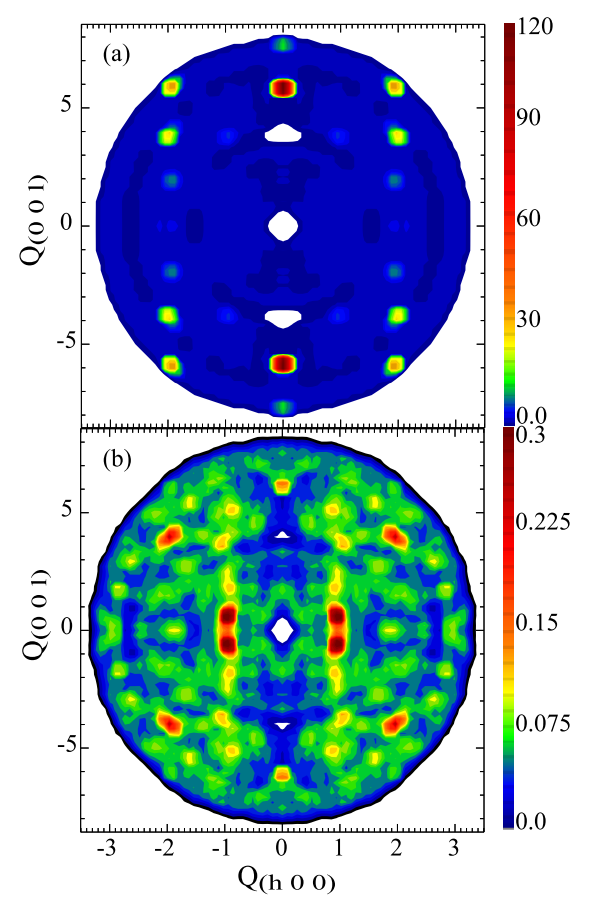

FIG. 1: (Color online) Neutron scattering intensity in the $(h, 0, l)$ plane of $\mathrm{La}_{2} \mathrm{CuO}_{4}$ at room temperature, i.e. just above the Néel temperature, measured using the multidetector on D7. Three-directional polarization analysis allows separation into (a) coherent structural scattering and (b) purely magnetic scattering, with removal of the incoherent background. A rod of magnetic scattering is developing along the $[1,0, l]$ direction showing the cross-over to $2 \mathrm{D}$ correlations.

Figure 3(a) shows that when data collected on D7 are corrected with a full knowledge of the spectral line shape they follow the same curve as those collected at fixed $\mathbf{Q}$ on IN20. Furthermore, the temperature dependence of intensities obtained using polarized neutrons is now in agreement with the predictions of the QNL $\sigma \mathrm{M}$. The insensitivity of linear spin-wave theory to four-particle terms means that the expansion of four-spin exchange operators in terms of gradients of the Néel vector does not add any new terms to the QNL $\sigma \mathrm{M}$. It is, therefore, gratifying that the clean measurements of the diffuse magnetic signal using polarized neutrons agree now with the predictions of the $\mathrm{QNL} \sigma \mathrm{M}$ for the renormalized-classical phase.
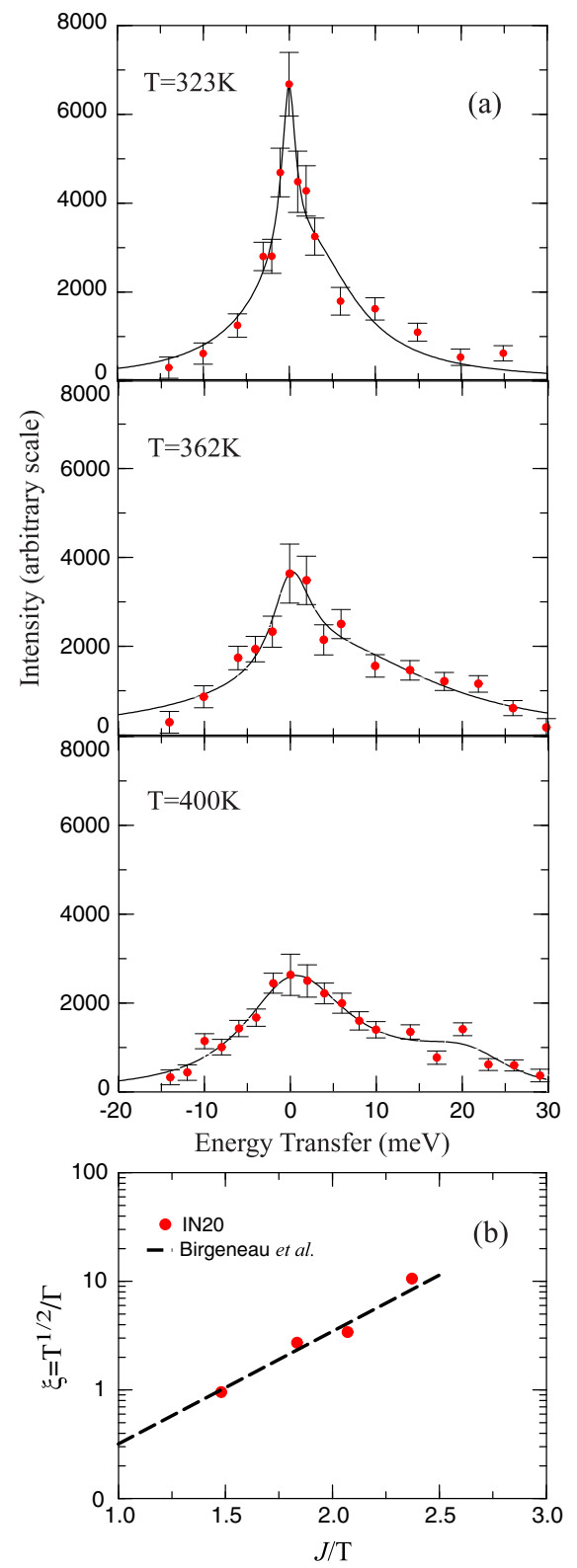

FIG. 2: (Color online) (a) Scans of energy transfer at fixed wave-vector transfer at several temperatures on IN20, and in (b) the characteristic energies are compared with the correlation lengths from Ref. 8] using the QNL $\sigma \mathrm{M}[9]$.

In Fig. 3 b) we show the comparison of the measured $S(0)$ with the results of a high-temperature series expansion of the multiple-spin exchange model Eq. (2). The high-temperature series expansions were taken to fifth order and analytically continued using biased Padé 

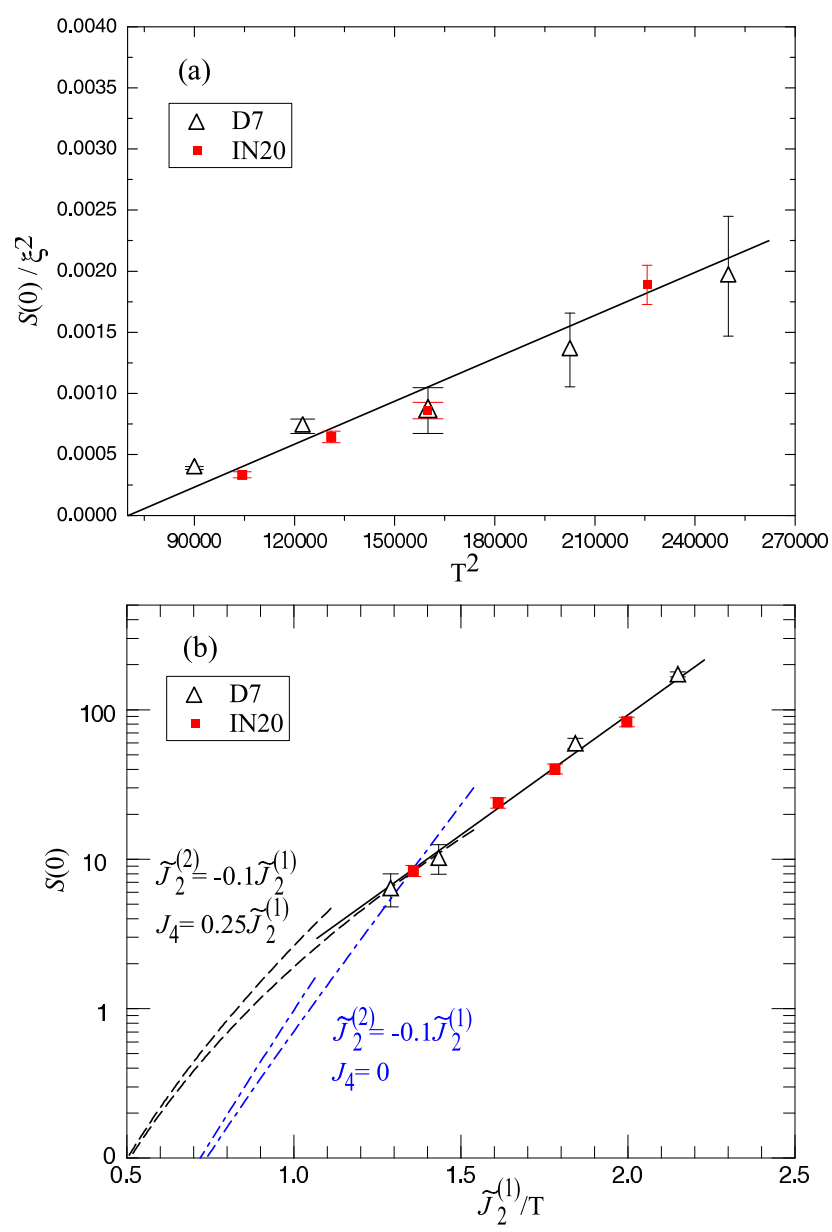

FIG. 3: (Color online) The neutron scattering amplitude $S(0)$ at $\mathbf{Q}=\left(\frac{1}{2}, \frac{1}{2}\right)$ of the cuprate square lattice. (a) The ratio of $S(0)$ over the square of the magnetic correlation length [8] varies linearly with temperature squared, in agreement with the QNL $\sigma \mathrm{M}[9]$. (b) The temperature dependence of $S(0)$ follows a straight (solid) line and the gradient agrees with the static susceptibility calculated in high-temperature series expansions with $J_{4}=0.25 \tilde{J}_{2}^{(1)}$ (dashed line). The dash-dot line shows the calculation with $J_{4}=0$. Fourth and fifth order expansions are shown, the latter extending to lower temperature.

approximants 15. The values of the pair exchange energies are those corresponding to the effective pair exchange $2 \widetilde{J}_{2}^{(1)}=111.8 \mathrm{meV}$ and $2 \tilde{J}_{2}^{(2)}=-11.4 \mathrm{meV}$ deduced from the magnon spectrum in the ordered phase [7]. Plotted on a semi log scale to extract the leading behaviour in $1 / T$, the experimental results fall on a straight line (solid line), and the gradient is in perfect agreement with the predictions of the series expansion at high temperatures with $J_{4}=0.25 \tilde{J}_{2}^{(1)}$ (dashed line) derived from the Hubbard model. This agreement is achieved with no free parameters except an overall scale factor. The dramatic difference in slope with respect to the dash-dot theoretical line (obtained with $J_{4}=0$ ) demonstrates the extreme sensitivity of the diffuse magnetic scattering to this term. These data constitute the first quantitative evidence for four-spin cyclic exchange in $\mathrm{La}_{2} \mathrm{CuO}_{4}$. We note that the ratio $J_{4} / \tilde{J}_{2}^{(1)} \approx 0.25$ is compatible with the predictions of the one-band Hubbard model, but more accurate neutron data would allow comparison with a more general three-band model $[13]$.

The higher order terms found to be of crucial importance in the physics of solid ${ }^{3} \mathrm{He}$ are also shown to be significant in an electronic magnetic material. It seems highly likely that ring exchange will be important in many other electronic magnetic systems, especially in those with strong hybridisation paths, such as the $\mathrm{Cu}_{4} \mathrm{O}_{4}$ plaquettes. Optical experiments indicate that higher order exchange is important in other hightemperature superconductors including $\mathrm{YBa}_{2} \mathrm{Cu}_{3} \mathrm{O}_{6.2}$, $\mathrm{Bi}_{2} \mathrm{Sr}_{2} \mathrm{Ca}_{0.5} \mathrm{Y}_{0.5} \mathrm{Cu}_{2} \mathrm{O}_{8+y}, \quad \mathrm{Nd}_{2} \mathrm{CuO}_{4}$ and $\mathrm{Pr}_{2} \mathrm{CuO}_{4}[4]$. The magnitude of the four-spin cyclic exchange is comparable to the pairing energies, and it is possible that circulating electronic currents have an important role in the mechanism of superconductivity. Ring exchange is also believed to be important in related ladder compounds, such as $\mathrm{La}_{6} \mathrm{Ca}_{8} \mathrm{Cu}_{24} \mathrm{O}_{41}$ and $\mathrm{Sr}_{14} \mathrm{Cu}_{24} \mathrm{O}_{41}$ 16.

In summary, four-spin cyclic exchange has been resolved in diffuse scattering experiments in the paramagnetic phase of $\mathrm{La}_{2} \mathrm{CuO}_{4}$, and the $2 \mathrm{D}$ critical fluctuations are correctly described by the QNL $\sigma \mathrm{M}$.

We would like to thank S.M. Hayden for the loan of the crystal and R. Coldea for helpful discussions. Financial support is gratefully acknowledged from the fifth European Community Framework Programme through contract HPRN-CT 2000-00166.

[1] W. Heisenberg, Z. Phys. 38, 411 (1926); ibid 49, 619 (1928).

[2] P.A.M. Dirac, Proc. Roy. Soc. A 123, 714 (1929).

[3] M. Roger, J.M Delrieu, and J.H. Hetherington, Rev. Mod. Phys. 55, 1 (1983).

[4] S. Sugai, et al. Phys. Rev. B 42, R1045 (1990).

[5] J.D. Perkins et al. Phys. Rev. Lett. 71, 1621 (1993).

[6] J. Lorenzana, J. Eroles and S. Sorella, Phys. Rev. Lett. 83, 5122 (1999).

[7] R. Coldea et al., Phys. Rev. Lett. 86, 5377 (2001).

[8] R. J. Birgeneau et al. Phys. Rev. B 59, 13788 (1999).

[9] S. Chakravarty, B.I. Halperin and D.R Nelson, Phys. Rev. B 39, 2344 (1989).

[10] D.J. Thouless, Proc. Phys. Soc. London 86, 893 (1965).

[11] M. Takahashi, J. Phys. C: Solid State Phys 10, 1289 (1977).

[12] M. Roger, cond-mat/0504141

[13] M. Roger and J.M Delrieu, Phys. Rev. B 39, 2299 (1989).

[14] O. Schärpf and H. Capellmann, Phys. Status Solidi A 135, 359 (1993).

[15] M. Roger, Phys. Rev. B 58, 11115 (1998).

[16] S. Brehmer et al. Phys. Rev. B 60, 329 (1999). 